\title{
[11C]-choline PET/CT' in imaging locally advanced prostate cancer
}

Marcello Rodari, Egesta Lopci, Giovanna Pepe,

Lidija Antunovic, Arturo Chiti

Nuclear Medicine, IRCCS Humanitas, Rozzano, Italy

[Received 15 IX 2011; Accepted 17 XI 2011] therapy, resulting in good PSA control. Serial bone scans during follow up were all negative for skeletal metastatic lesions. Six years later, routine controls showed rising PSA levels $(6.83 \mathrm{ng} / \mathrm{ml})$ not responding to maximal androgen blockade (MAB). The referring clinician required a $[11 \mathrm{C}]$-choline PET/CT for patient restaging before intensity modulated radiation therapy (IMRT).

PET/CT was performed 10 minutes after IV injection of 360 $\mathrm{MBq}$ of [11C]-choline, on a Siemens Biograph 6 LSO scanner. PET images demonstrated an increased [11C]-choline uptake in the prostate gland extending to the adjacent tissues. The early image acquisition and the delayed urinary excretion of $[11 \mathrm{C}]$-choline permitted the detection of bladder wall infiltration (Figures 1 and 2).

Since its first introduction in 1997 [1], PET Imaging with [11C]choline has become a useful tool in the investigation of prostate cancer, with its main application in previously treated patients with rising PSA serum levels and negative conventional imaging procedures [2]. Its diagnostic capability is not only limited to loco regional lymph nodes and distant bone metastases, but also in the detection of local disease [2, 3].

However, because of the short half-life of carbon-11 (T1/2 $20 \mathrm{~min}$ ), requiring on site cyclotron production, the [18F]-labelled analogue (18F-fluorocholine) was developed and introduced in clinical practice with similar applications [4]. No significant differences are reported in the two tracer distributions except for an early urinary appearance of [18F]-fluorocholine in the urinary tract due to an incomplete tubular reabsorption [5]. This excretion does not affect the overall diagnostic accuracy of [18F]-choline PET, but can be a drawback when considering local recurrence. Compared with [18F]-choline, [11C]-choline has a negligible urinary elimination which, in selected cases, proves to be an advantage. Nonetheless, dedicated protocols to minimize the influence of bladder activity can be easily implemented and, whatever the radiopharmaceutical used, we recommend obtaining an empty bladder when evaluating prostate cancer patients. 
A
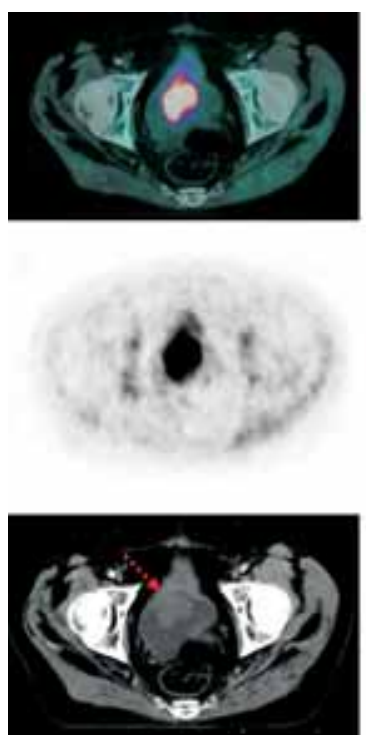

B

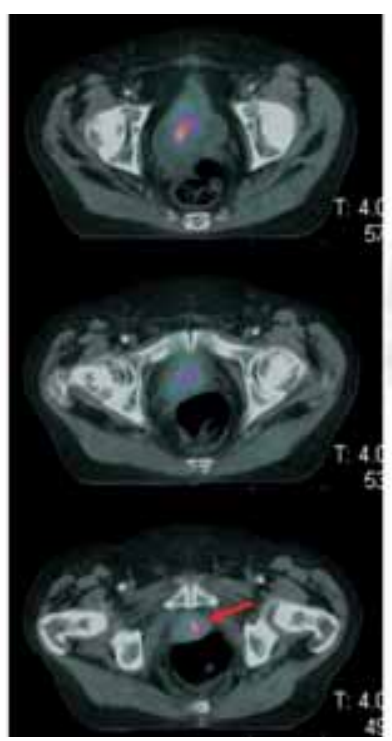

MIP image

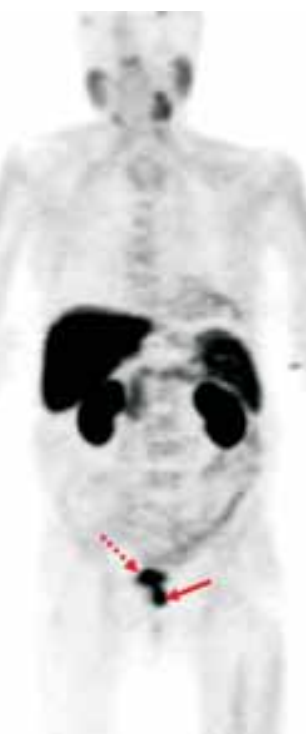

Figure 1. Axial and MIP images of [11C]-choline PET/CT scan demonstrating bladder wall invasion (dashed arrows) (A), along with persisting disease in the prostate gland (straight arrows) (B).

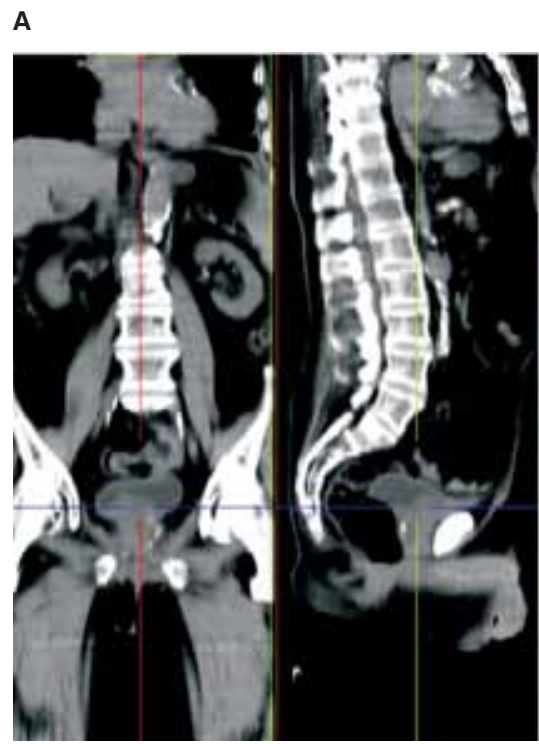

B

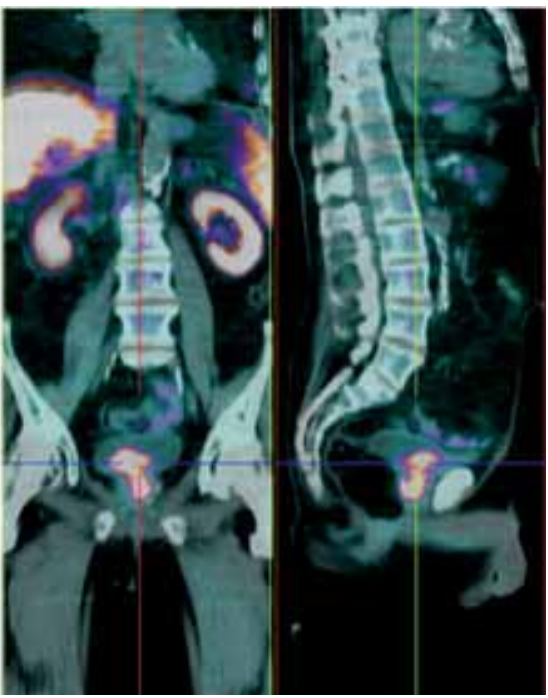

Figure 2. Coronal and sagittal low dose CT (A) and fused PET/CT images (B) showing the full extent of neoplastic disease interesting the prostate gland up to the inferior bladder wall.

\section{References}

1. Hara T, Yuasa M, Yoshida H. Automated synthesis of fluorine-18 labeled choline analogue: 2-fluoroethyl- dimethyl-2-oxyethylammonium [abstract]. J Nucl Med 1997; 38: 44P.

2. Picchio M, Giovannini E, Crivellaro $C$ et al. Clinical evidence on PET/CT for radiation therapy planning in prostate cancer. Radiother Oncol 2010; 96: 347-350

3. Jadvar H. Prostate cancer: PET with 18 F-FDG, 18 F- or $11 \mathrm{C}$-acetate and 18F- or 11C-choline. J Nucl Med 2011; 52: 81-89

4. DeGrado TR, Coleman RE, Wang $S$ et al. Synthesis and evaluation of $18 \mathrm{~F}$ labeled choline as an oncologic tracer for positron emission tomography: Initial findings in prostate cancer. Cancer Res 2001; 61: 110-117.

5. Husarik DB, Miralbell R, Dubs M et al. Evaluation of [(18)F]-choline $\mathrm{PET} / \mathrm{CT}$ for staging and restaging of prostate cancer. Eur $\mathrm{J}$ Nucl Med Mol Imaging 2008; 35: 253-263. 